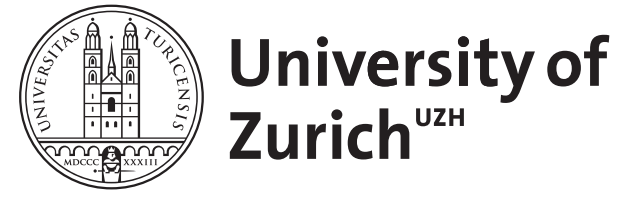

\title{
Gastroenterologische Notfälle
}

Gundling, F ; Fried, M ; Schepp, W

DOI: https://doi.org/10.1007/s11377-011-0575-4

Posted at the Zurich Open Repository and Archive, University of Zurich ZORA URL: https://doi.org/10.5167/uzh-156025

Journal Article

Published Version

Originally published at:

Gundling, F; Fried, M; Schepp, W (2012). Gastroenterologische Notfälle. Der Gastroenterologe, 7(2):8586.

DOI: https://doi.org/10.1007/s11377-011-0575-4 
Gastroenterologe 2012 $\cdot 7: 85-86$

DOI 10.1007/s11377-011-0575-4

๑) Springer-Verlag 2012

\section{F. Gundling ${ }^{1} \cdot$ M. Fried ${ }^{2} \cdot$ W. Schepp ${ }^{1}$}

${ }^{1}$ Klinik für Gastroenterologie, Hepatologie und Gastroenterologische

Onkologie, Klinikum Bogenhausen, Städtisches Klinikum München

${ }^{2}$ Abteilung Gastroenterologie und Hepatologie, UniversitätsSpital Zürich

\section{Gastroenterologische Notfälle}

Für zahlreiche Teilgebiete der inneren Medizin wie z. B. Kardiologie und Intensivmedizin sind Diagnostik und Therapie der wichtigsten Notfallsituationen durch Leitlinien klar definiert; erinnert sei exemplarisch an die kardiopulmonale Reanimation oder das akute Koronarsyndrom. Dagegen existieren ähnlich klare Vorgaben für weitaus weniger gastroenterologische Notfälle. Manche dieser Empfehlungen sind darüber hinaus veraltet, ungeachtet zahlreicher neuer klinischer Studien. Die aktuellsten deutschen Leitlinien zur akuten Pankreatitis stammen noch aus dem Jahr 2000, obwohl im amerikanisch-angelsächsischen Sprachraum mittlerweile aktuellere Empfehlungen vorliegen $[2,1]$.

Manche gastroenterologischen Notfälle weisen hinsichtlich ihrer Ätiologie und Prognose eine große Spannweite auf. Dies erschwert die Etablierung fester Behandlungsalgorithmen. Die erfolgreiche Behandlung gastroenterologischer Notfälle setzt daher häufig neben Kenntnissen der aktuellen medizinischen Literatur und Datenlage auch ein gewisses „Bauchgefühl“ voraus. Dieses berücksichtigt eben nicht nur Evidenz, sondern auch den klinischen Erfahrungsschatz. Dies gilt für die verschiedensten gastroenterologischen Notfälle:

- Der Ileus als klinische Endstrecke zahlreicher ätiologisch unterschiedlichster Krankheitsbilder reicht von der oft harmlosen, klinisch kompensierten Behinderung des gastrointestinalen Transports („Subileus“) bis zu vital bedrohlichen Komplikationen wie Darmischämie, Perforation und Ileuskrankheit. Die letzteren sind mit einer erheblichen Mortalität verbunden.

- Die meisten Fälle einer akuten Pankreatitis verlaufen prognostisch günstig. In selteneren Fällen kann jedoch eine akute Bauchspeicheldrüsenentzündung auch einen schweren, möglicherweise letalen Verlauf nehmen.

Die richtige und vor allem frühzeitige Einschätzung des Schweregrades eines Krankheitsbildes ist insbesondere in der Gastroenterologie wichtig, um zwischen einem gravierenden Notfall und einer dringlichen, aber nicht lebensbedrohlichen Diagnose zu unterscheiden. Auch gibt es bei den meisten gastroenterologischen Notfällen nur selten „unbestechliche", richtungsweisende Laborparameter, die das ganze Ausmaß der Erkrankung frühzeitig erkennen lassen - wie z. B. die Hämoglobinkonzentration bei der gastrointestinalen Blutung. Während die Höhe der myokardialen Nekroseparameter im Serum früh Aufschlüsse über den Schweregrad eines Herzinfarkts geben kann, schließt auch eine initial nur gering erhöhte Amylase- oder Lipaseaktivität im Serum einen schweren späteren Verlauf einer Pankreatitis nicht aus.

Eine besondere Gefahr gastroenterologischer Notfallsituationen stellt daher die zeitliche Verzögerung entsprechender diagnostischer und therapeutischer Maßnahmen dar. Dank gängiger Slogans wie „time is muscle" oder "time is brain" ist inzwischen jedem Arzt in der Notaufnahme die Bedeutung des Zeitfaktors für die Akutversorgung von akutem Koronarsyndrom oder Schlaganfall bekannt: Je rascher die Reperfusion erreicht wird, desto weniger Myokard bzw. Gehirn gehen verloren. Idealerweise bleibt die Erkrankung dann ohne wesentliche Schäden, eine Wiedereingliederung in das soziale Umfeld und in den normalen Arbeitsprozess ist möglich.

> Auch bei der Akutversorgung von Patienten in gastroenterologischen Notfallsituationen ist häufig die Zeit der wichtigste Faktor.

So entscheidet sich die Prognose der akuten Pankreatitis oft in den ersten 48-72 Stunden [3]. Die Dünndarmmukosa toleriert eine Ischämie nur für etwa 6 Stunden. Man könnte die oben erwähnten Schlagworte also getrost um die Maxime „time is bowel“ erweitern.

Die vorliegende Ausgabe von Der Gastroenterologe widmet sich daher dem Thema „Gastroenterologische Notfälle“. Namhafte Autoren und Experten berichten über wichtige Krankheitsbilder, die im klinischen gastroenterologischen Alltag häufig vorkommen oder zumindest häufig in die differenzialdiagnostischen Erwägungen einbezogen werden müssen. Die Herausgeber würden sich daher freuen, wenn die Beiträge schon aufgrund ihrer großen Relevanz auf das Interesse einer breiten Leserschaft stoßen würden, zumal das Spektrum der Themen über die Gastroenterologie hinaus von der Viszeralchirurgie bis in die Notfallmedizin reicht.
F. Gundling
M. Fried
W. Schepp 


\section{Korrespondenzadresse

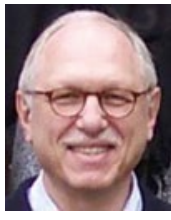 \\ Prof. Dr. M. Fried \\ Abteilung Gastroenterologie \\ und Hepatologie, \\ UniversitätsSpital Zürich \\ Rämistraße 100, 8091 Zürich \\ Schweiz \\ michael.fried@usz.ch

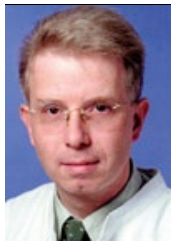 \\ Prof. Dr. W. Schepp \\ Klinik für Gastroenterologie, \\ Hepatologie und Gastro- \\ enterologische Onkologie, \\ Klinikum Bogenhausen, \\ Städtisches Klinikum München \\ Englschalkinger Straße 77, \\ 81925 München \\ gastroenterologie.kb@ \\ klinikum-muenchen.de}

\section{Literatur}

1. Rünzi M, Layer P, Büchler MW et al. (2000) Therapie der akuten Pankreatitis. Gemeinsame Leitlinien. Z Gastroenterol 38: 571-581

2. Forsmark CE, Baillie J (2007) AGA institute techical review on acute pancreatitis. Gastroenterology 132: 2022-2044

3. McKay CJ, Imrie CW (2004) The continuing challenge of early mortality in acute pancreatitis. Br J Surg 91: 1243-1244

\section{Ausschreibung Oskar Medizin-Preis 2012}

Die Stiftung Oskar-Helene-Heim, deren Zweck die Förderung der Wissenschaft und Forschung ist, verleiht jährlich den mit $\mathbf{5 0 . 0 0 0 €}$ dotierten Oskar Medizin-Preis.

Der Preis wird im Jahr 2012 auf den Gebieten der

\section{Gastroenterologie/Viszeralchirurgie}

ausgeschrieben. Die Therapie von Magenund Darmerkrankungen, die durch das zentrale und enterische Nervensystem verursacht bzw. beeinflusst sind, stellen eine schwerwiegende Problematik dar. Mit dem Preis soll daher ein habilitierter Mediziner ausgezeichnet werden, der zum Thema

\section{„Einfluss des zentralen und enterischen Nervensystems auf Erkrankungen und Funktionen im Magen-Darmbereich"}

besonders relevante Ergebnisse der Grundlagen- und/oder klinischen Forschung in Deutschland erzielt hat. Fachübergreifende Arbeitsgruppen sind ebenfalls zur Bewerbung zugelassen.

Der prämierte Erkenntnisgewinn soll einer breiten Öffentlichkeit vermittelbar sein. Dem Antrag sollen der Lebenslauf, die 5 wichtigsten Arbeiten aus den letzten 3 Jahren zum Thema „Einfluss des zentralen und enterischen Nervensystems auf MagenDarmerkrankungen" sowie eine inhaltliche Zusammenfassung der Forschungsergebnisse beigefügt werden.

Das Preisgeld ist für Forschungszwecke nach der freien Entscheidung des Preisträgers zu verwenden. Der Preisträger hat der Stiftung diese Verwendung in geeigneter Weise zu belegen.

Die Preisverleihung wird im letzten Quartal 2012 in Berlin erfolgen. Der Antrag ist bis zum 30. Juni 2012 bei der Stiftung Oskar-Helene-Heim einzureichen (www.stiftung-ohh.de).

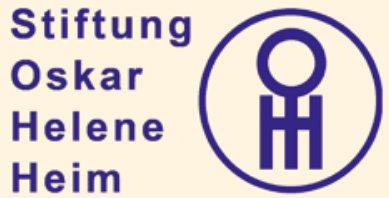

Weitere Informationen erteilt der Geschäftsführer der Stiftung, Werner Ukas, werner.ukas@stiftung-ohh.de oder info@stiftung-ohh.de, Tel.: 030/8102-1100

Die Schirmherrschaft für diesen Preis hat die Bundesministerin für Bildung und Forschung, Frau Prof. Dr. Annette Schavan, übernommen. Mit diesem Medizinpreis sollen hervorragende Leistungen, insbesondere auf einem der folgenden Gebiete gewürdigt werden: Orthopädie und Orthopädie-Technik, Pneumologie sowie Gastroenterologie/Viszeralchirurgie.

Quelle: Stiftung Oskar-Helene-Heim, www.stiftung-ohh.de 